\title{
Combined Adenosquamous and Large Cell Neuroendocrine Carcinoma of the Gallbladder
}

Jiyoon Jung · Yang-Seok Chae Chul Hwan Kim • Youngseok Lee Jeong Hyeon Lee · Dong-Sik Kim ${ }^{1}$ Young-Dong $\mathrm{Yu}^{1} \cdot$ Joo Young Kim

Departments of Pathology and ${ }^{1}$ Surgery, Korea University Anam Hospital, Korea University

College of Medicine, Seoul, Korea

Received: June 29, 2017

Revised: August 1, 2017

Accepted: August 20, 2017

\section{Corresponding Author}

Joo Young Kim, MD, PhD

Department of Pathology, Korea University Anam Hospital, Korea University College of Medicine, 73 Inchon-ro, Seongbuk-gu, Seoul 02841, Korea Tel: +82-2-920-6268

Fax: $+82-2-920-6576$

E-mail: lepetit80@hanmail.net
Large cell neuroendocrine carcinoma (LCNEC) of the gallbladder is extremely rare and usually combined with other type of malignancy, mostly adenocarcinoma. We report an unusual case of combined adenosquamous carcinoma and LCNEC of the gallbladder in a 54-year-old woman. A radical cholecystectomy specimen revealed a $4.3 \times 4.0 \mathrm{~cm}$ polypoid mass in the fundus with infiltration of adjacent liver parenchyma. Microscopically, the tumor consisted of two distinct components. Adenosquamous carcinoma was predominant and abrupt transition from adenocarcinoma to squamous cell carcinoma was observed. LCNEC showed round cells with large, vesicular nuclei, abundant mitotic figures, and occasional pseudorosette formation. The patient received adjuvant chemotherapy. However, multiple liver metastases were identified at 3-month follow-up. Metastatic nodules were composed of LCNEC and squamous cell carcinoma components. Detecting LCNEC component is important in gallbladder cancer, because the tumor may require a different chemotherapy regimen and show early metastasis and poor prognosis.

Key Words: Carcinoma, adenosquamous; Large cell neuroendocrine carcinoma; Gallbladder; Prognosis
Large cell neuroendocrine carcinoma (LCNEC) of the gallbladder is an extremely rare malignancy, comprising $2 \%$ of all gallbladder cancers. ${ }^{1}$ The prognosis of this tumor is poor and early metastasis is common. ${ }^{2}$ To date, 18 cases of gallbladder LCNEC have been reported. ${ }^{3,4}$ LCNEC of the gallbladder is usually combined with other types of malignancies, mostly adenocarcinoma. We present an unusual case of combined adenosquamous carcinoma (ASC) and LCNEC of the gallbladder.

\section{CASE REPORT}

A 54-year-old woman presented with a 4-month history of intermittent epigastric pain. Laboratory tests revealed elevated serum levels of alkaline phosphatase and gamma-glutamyltransferase, up to $161 \mathrm{IU} / \mathrm{L}$ (reference range, 30 to $120 \mathrm{IU} / \mathrm{L}$ ) and 81 $\mathrm{IU} / \mathrm{L}$ (reference range, 9 to $64 \mathrm{IU} / \mathrm{L}$ ), respectively. The levels of other liver enzymes including aspartate aminotransferase, alanine aminotransferase, as well as total bilirubin and direct bilirubin levels, were within normal range. Serum carbohydrate-associated antigen (CA19-9) was $151.4 \mathrm{IU} / \mathrm{mL}$ (normal < $37 \mathrm{IU} / \mathrm{mL}$ ), and $\alpha$-fetoprotein and carcinoembryonic antigen were within normal range. Abdominal computed tomography showed a $6.9 \mathrm{~cm}$ mass in the gallbladder fundus, with direct invasion of the adjacent liver (Fig. 1A). Biopsy of the liver mass showed poorly differentiated carcinoma with extensive squamous differentiation.

Radical cholecystectomy was performed and gross examination revealed a $4.3 \times 4.0 \mathrm{~cm}$ polypoid mass in the gallbladder fundus with infiltration of the adjacent liver parenchyma and multiple yellowish gallstones (Fig. 1B, C). An incidentally found $0.4-\mathrm{cm}-$ sized nodule was also noted in the liver.

Microscopically, the tumor comprised two distinct components: ASC and LCNEC. The adenocarcinoma (AC) component of the ASC consisted of variable sized glands, lined by atypical columnar epithelium. The squamous cell carcinoma (SCC) component showed atypical stratified squamous epithelium with occasional keratin pearls. An abrupt transition from AC to SCC was observed (Fig. 2A, B). In addition, there was an area of LCNEC consisting of round cells with large, vesicular nuclei and frequent mitotic figures (33/10 high-power fields). Occasional rosette formation was found. The tumor cells were arranged in trabecular, palisad- 
ing, or solid patterns, and mixed with an $\mathrm{AC}$ component in the periphery (Fig. 2C, D). These cells showed diffuse and strong positivity for synaptophysin and chromogranin, and focal positivity for CD56 immunohistochemistry (Fig. 3A-C). The Ki-67 proliferating labeling index was 25\% (Fig. 3D). The tumor was largely composed of ASC, and the proportion of the SCC component was approximately $80 \%$, whereas the area of LCNEC was small, constituting approximately $10 \%$ of the entire tumor. The gallbladder wall was mainly composed of AC components, while the proportion of SCC and LCNEC components increased substantially as the tumor invaded into the liver parenchyma. Multifocal lymphovascular invasion by SCC and perineural tumor invasion by LCNEC were identified. Only a few foci of lymphovascular invasion were observed with an AC component. Chronic cholecystitis with intestinal metaplasia were identified in the remaining mucosa. No metastasis was present in the seven regional
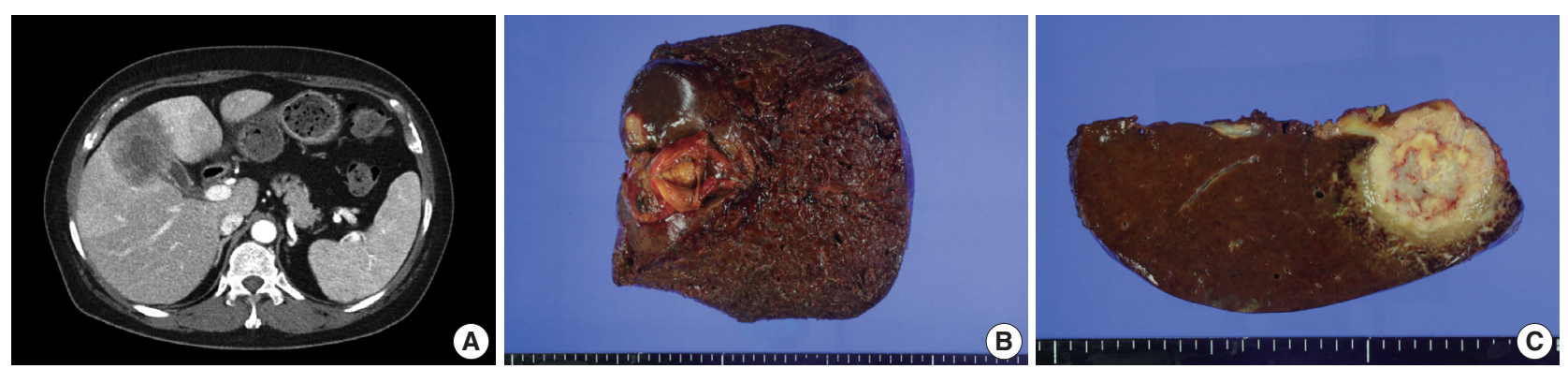

Fig. 1. Imaging and gross findings. (A) On abdominal computed tomography, the tumor of the gallbladder shows direct invasion of the adjacent liver. (B) On opening the gallbladder, a fungating mass is observed in the fundus. (C) The cut surface shows a relatively well-circumscribed, yellowish fibrotic mass, with hemorrhage and necrosis, invading the liver parenchyma.
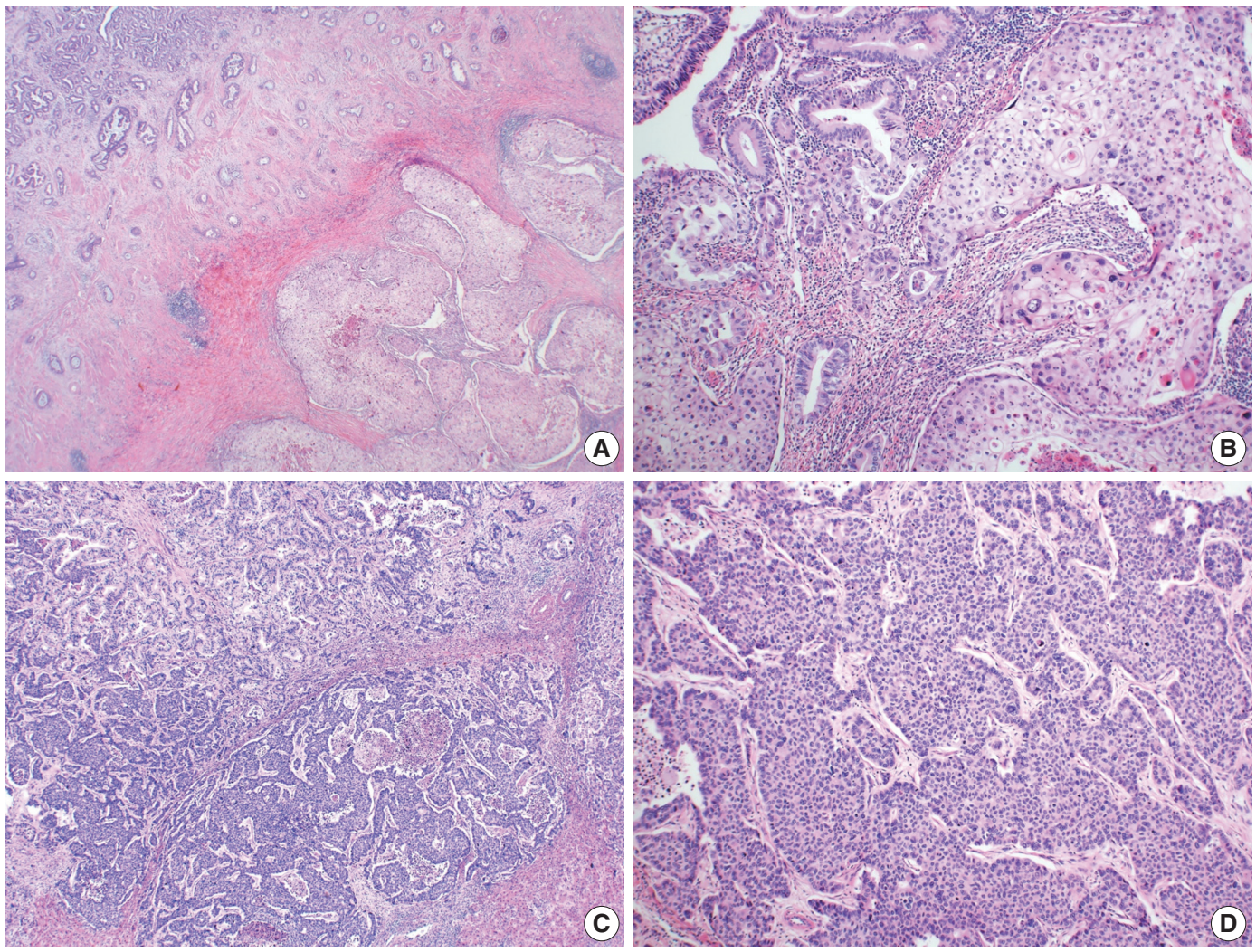

Fig. 2. Microscopic findings. (A, B) Adenosquamous carcinoma component consisted of variable-sized glands lined by atypical columnar epithelium (left) and atypical stratified squamous epithelium (right). (C) Neuroendocrine carcinoma component, transition from adenocarcinomatous component (left) and composed of large cells arranged in solid sheets (right). (D) The neuroendocrine component shows solid and pseudorosette patterns. 

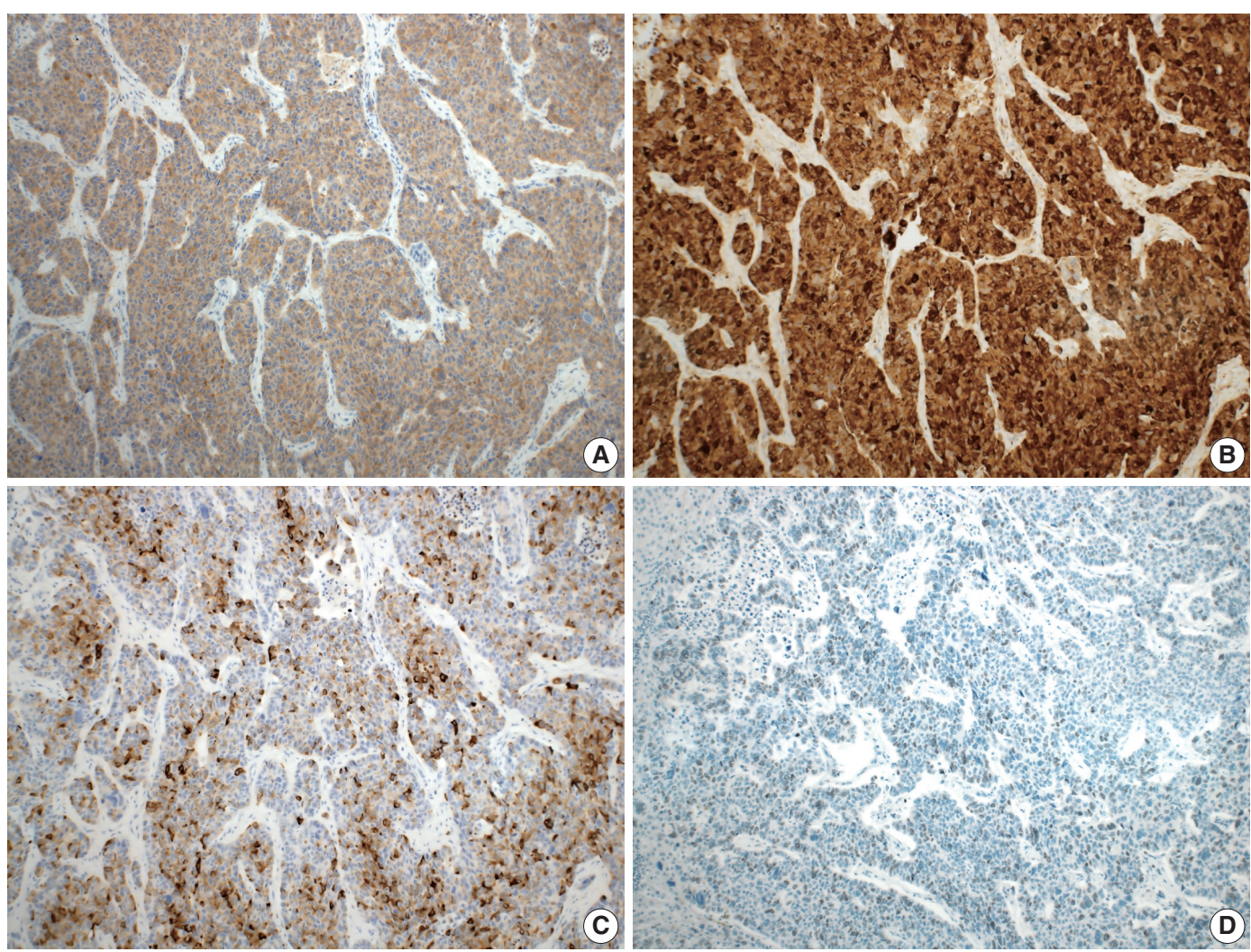

Fig. 3. Immunohistochemical findings. Large cell neuroendocrine carcinoma component was diffusely positive for synaptophysin (A), and chromogranin (B), and focal positive for CD56 (C). (D) The Ki-67 proliferating index was up to $25 \%$.

lymph nodes. The metastatic nodule in the liver was composed purely of an LCNEC component. The final diagnosis of the gallbladder was confirmed as combined ASC and LCNEC. The patient received adjuvant chemotherapy post-operatively. However, magnetic resonance imaging revealed two liver metastases at the 3-month follow-up. Wedge resection of the liver was performed on the metastatic nodules, measuring $4.7 \mathrm{~cm}$ and 3.4 $\mathrm{cm}$. Microscopically, the metastatic nodules were composed purely of an SCC component with no AC or LCNEC components. The patient was treated with chemotherapy (gemcitabine, cisplatin, and epirubicin) and radiotherapy after the operation, and died after 13 months from the initial diagnosis.

This study was approved by the Institutional Review Board of Korea University Anam Hospital, and informed consent was waived (ED17132).

\section{DISCUSSION}

The majority of carcinomas in the gallbladder are ACs, comprising up to $88 \%$. Other carcinomas include ASC (5\%), neuroendocrine carcinoma (NEC) (2\%), SCC (1\%), and undifferentiated carci- noma $(<1 \%))^{5}$ Identifying the exact histologic component of the tumor is important because the histologic type is associated with patient prognosis in gallbladder cancers. ACs have a relatively favorable prognosis, whereas tumors with squamous or neuroendocrine differentiation have a poor outcome (mean survival time, 50 vs 23 or 10 months, respectively). ${ }^{2,6}$ LCNEC has been known to exhibit aggressive behavior and early metastasis. ${ }^{4}$ Among the previously reported LCNEC cases, $53 \%$ had directly invaded the liver at the time of diagnosis, and $47 \%$ had been identified with locoregional or distant metastases involving bone, liver or lymph nodes. ${ }^{3}$ In this case, the tumor showed direct invasion of the liver parenchyma at the time of initial diagnosis and additional multiple liver metastases afterwards, demonstrating aggressive behavior.

According to the recent 2010 World Health Organization classification, neoplasms, morphologically composed of both glandular epithelial cells and neuroendocrine cells with over 30\% of each component are described as mixed adenoneuroendocrine carcinoma (MANEC). ${ }^{7}$ The present case was predominantly composed of SCC (about 80\%) and the amount of AC or NEC component was not enough for the criteria of MANEC. 
The histogenesis of NEC in the gallbladder is still not clear, because neuroendocrine cells are not normally present in the gallbladder except for a few cells in the neck region. It has been postulated that they may arise from pluripotent precursor cells. ${ }^{8}$ However, the pathogenesis of differentiation from stem cells to neuroendocrine cells remains unclear. Another hypothesis for the development of NEC of the gallbladder is through metaplastic change. ${ }^{9}$ Intestinal or gastric metaplasia occurs as a result of cholelithiasis and chronic inflammation. ${ }^{10}$ Argentaffin cells have been identified in the metaplastic mucosa and these cells have been suggested as precursors of neuroendocrine tumor in the gallbladder. ${ }^{11}$ Almost all previously reported gallbladder LCNECs described coexisting cholelithiasis with chronic cholecystitis. ${ }^{5}$ In this case, chronic cholecystitis with intestinal metaplasia associated with cholelithiasis was identified, which suggested a precursor lesion of the NEC.

It is noteworthy that metastatic nodules show two different histologic types. The incidentally identified, synchronous metastatic hepatic nodule was totally composed of an LCNEC component, whereas the metachronous metastatic nodules were composed entirely of an SCC component. The metastatic potential is different in each type of cancer cells. ${ }^{12}$ The SCC component has higher proliferative activity than the $\mathrm{AC}$ component and more frequently observed in the invasive front of the tumor and in lymphovascular and perineural invasion in the gallbladder ASC. ${ }^{13}$ This might contribute to the metastatic potential and tumor progression in ASC. ${ }^{13}$ In the present case, the SCC component was frequently observed in lymphovascular invasion and caused multiple pure SCC metastatic nodules. Additionally, LCNEC has higher risk of metastasis through lymphatic invasion, compared to the AC. ${ }^{14}$ Acosta and Wiley ${ }^{15}$ reported that the majority of lymph node metastases shows only NEC components in primary MANECs of the biliary tree. This case is consistent with previous studies in that the metastatic nodules were composed of either SCC or NEC, without an AC component, and this might contribute to the metastatic potential and poor prognosis of ASC and NEC of the gallbladder.

There are also differences in chemotherapeutic agents between conventional AC and combined tumor of the gallbladder. Conventional $A C$ is usually treated with gemcitabine and cisplantin, while combined ASC or NEC is treated with FOLFOX (leucovorin, fluorouracil, and oxaliplatin) or etoposide. ${ }^{4,16}$

This is an unusual case of LCNEC combined with ASC. The histologic types of the gallbladder carcinoma might contribute to the metastatic potential and poor prognosis. To detect an LCNEC or ASC component is important because the tumor may require a different chemotherapy regimen and show early metastasis with poor prognosis.

\section{Conflicts of Interest}

No potential conflict of interest relevant to this article was reported.

\section{REFERENCES}

1. National Cancer Institute. SEER data, 1973-2005 [Internet]. Rockville: National Cancer Institute, 2008 [cited 2017 Sep 1]. Available from: http://seer.cancer.gov/.

2. Henson DE, Albores-Saavedra J, Corle D. Carcinoma of the gallbladder: histologic types, stage of disease, grade, and survival rates. Cancer 1992; 70: 1493-7.

3. Liu W, Wang L, He XD, Feng C, Chang XY, Lu ZH. Mixed large cell neuroendocrine carcinoma and adenocarcinoma of the gallbladder: a case report and brief review of the literature. World J Surg Oncol 2015; 13: 114.

4. Buscemi S, Orlando E, Damiano G, et al. "Pure" large cell neuroendocrine carcinoma of the gallbladder: report of a case and review of the literature. Int J Surg 2016; 28 Suppl 1: S128-32.

5. Eltawil KM, Gustafsson BI, Kidd M, Modlin IM. Neuroendocrine tumors of the gallbladder: an evaluation and reassessment of management strategy. J Clin Gastroenterol 2010; 44: 687-95.

6. Alpuerto AC, Mora ME, Robitsek RJ, Schubl SD. Primary pure squamous cell carcinoma of the gallbladder locally invading the liver, duodenum, and stomach: a case report and literature review. Case Rep Surg 2017; 2017: 2534029.

7. Bosman FT, Carneiro F, Hruban RH, Theise ND. WHO classification of tumours of the digestive system. 4th ed. Lyon: IARC Press, 2010; 274-6.

8. Noske A, Pahl S. Combined adenosquamous and large-cell neuroendocrine carcinoma of the gallbladder. Virchows Arch 2006; 449: 135-6.

9. Kanthan R, Senger JL, Ahmed S, Kanthan SC. Gallbladder cancer in the 21st century. J Oncol 2015; 2015: 967472.

10. Albores-Saavedra J, Nadji M, Henson DE, Angeles-Angeles A. Enteroendocrine cell differentiation in carcinomas of the gallbladder and mucinous cystadenocarcinomas of the pancreas. Pathol Res Pract 1988; 183: 169-75.

11. Christie AC. Three cases illustrating the presence of argentaffin (kultschitzky) cells in the human gall-bladder. J Clin Pathol 1954; 7 318-21.

12. Hart IR, Fidler IJ. The implications of tumor heterogeneity for studies 
on the biology of cancer metastasis. Biochim Biophys Acta 1981; 651:37-50.

13. Hoshimoto S, Hoshi S, Hishinuma S, et al. Adenosquamous carcinoma in the biliary tract: association of the proliferative ability of the squamous component with its proportion and tumor progression. Scand J Gastroenterol 2017; 52: 425-30.

14. Chen C, Wang L, Liu X, Zhang G, Zhao Y, Geng Z. Gallbladder neuroendocrine carcinoma: report of 10 cases and comparision of clinicopathologic features with gallbladder adenocarcinoma. Int J Clin Exp Pathol 2015; 8: 8218-26.

15. Acosta AM, Wiley EL. Primary biliary mixed adenoneuroendocrine carcinoma (MANEC): a short review. Arch Pathol Lab Med 2016; 140: 1157-62.

16. Verlicchi L, Blons H, Hannoun L, Bachet JB. Squamous-cell gallbladder carcinoma: how to treat? J Cell Sci Ther 2015; 6: 212. 